\title{
Stray light analysis for the Thomson scattering diagnostic of the ETE Tokamak
}

\author{
L. A. Berni ${ }^{1}$ and B. F. C. Albuquerque ${ }^{2}$ \\ ${ }^{1}$ Instituto Nacional de Pesquisas Espaciais (INPE), Laboratório Associado de Sensores e Materiais (LAS), \\ 12.227-010 São José dos Campos, SP, Brazil \\ ${ }^{2}$ Instituto Nacional de Pesquisas Espaciais (INPE), Engenharia e Tecnologia Espaciais, Divisão de Eletrônica \\ Aeroespacial, 12.227-010 São José dos Campos, SP, Brazil
}

(Received 31 May 2010; accepted 27 September 2010; published online 14 December 2010)

\begin{abstract}
Thomson scattering is a well-established diagnostic for measuring local electron temperature and density in fusion plasma, but this technique is particularly difficult to implement due to stray light that can easily mask the scattered signal from plasma. To mitigate this problem in the multipoint Thomson scattering system implemented at the ETE (Experimento Tokamak Esférico) a detailed stray light analysis was performed. The diagnostic system was simulated in ZEMAX software and scattering profiles of the mechanical parts were measured in the laboratory in order to have near realistic results. From simulation, it was possible to identify the main points that contribute to the stray signals and changes in the dump were implemented reducing the stray light signals up to 60 times. (C) 2010 American Institute of Physics. [doi:10.1063/1.3505485]
\end{abstract}

\section{INTRODUCTION}

Thomson scattering is a well-established diagnostic for measuring local electron temperature and density in fusion plasma experiments with spatial and temporal resolution. ${ }^{1}$ This technique is particularly difficult to implement due to the extremely low ratio between the scattered signal and laser power that probes the plasma. Usually this ratio is in the range of $10^{-14}$ and the laser power is of several MW. For this reason, the scattered signals can be easily masked by the laser stray light as well as by the plasma background radiation. This work proposes a detailed stray light analysis of the diagnostic installed at the ETE (Experimento Tokamak Esférico). ${ }^{2,3}$ The diagnostic was simulated in ZEMAX software where the scattering profiles used for the mechanical surfaces involved (steel, inconel, aluminum, and graphite) were measured in laboratory in order to have near realistic results. With this simulation it was possible to identify and quantify the main points that contribute to the stray signals and changes in the system were implemented to reduce the stray light.

\section{MULTIPOINT THOMSON SCATTERING DIAGNOSTIC OF THE ETE TOKAMAK}

In order to measure the electron temperature and density of the plasma a Multipoint Thomson Scattering system was implemented in the ETE Tokamak. Figure 1 shows a top view of the diagnostic setup with details of the laser beam path. This system is based on a $10 \mathrm{~J}$ Q-switched ruby laser that is operated in single pulse mode per plasma discharge. A set of three flat mirrors guide the laser beam to the entrance window and a $3 \mathrm{~m}$ focal lens focuses the beam in the middle of the plasma column. The entrance window is connected to the vacuum vessel through a $1.38 \mathrm{~m}$ long flight tube. Inside this tube there are ten vanes to minimize the stray light. The first vane $(\Phi=25 \mathrm{~mm})$ is placed near the window and at the end of the tube there is a $20 \mathrm{~mm}$ diameter vane. The other eight vanes are distributed along the tube length. Inside the dump there are two big vanes $(\Phi=40 \mathrm{~mm})$ and two graphite plates to stop the laser beam. A specially designed objective lens collects the scattered light from plasma along the beam path that is imaged on a set of ten mono fibers with a core diameter of $0.8 \mathrm{~mm}$ and $\mathrm{NA}=0.39$. In order to match the objective $(\mathrm{NA}=0.08)$ and the fibers numerical apertures, and to enlarge the observation region to $4 \mathrm{~mm}$, microlenses are used $(f=15 \mathrm{~mm}$ and $\phi=3 \mathrm{~mm})$ in front of each fiber. The fiber holder at the back of the objective presents 23 fixed positions for the fibers that allows the observation from $R=15 \mathrm{~cm}$ to $R=55 \mathrm{~cm}$ along the laser beam path inside the plasma column. Each fiber has different lengths (time delay line) to relay the scattered light signals to the same detector. ${ }^{2,3}$ The scattered light by the plasma is detected by a polychromator that presents four channels for temperature measurements. The polychromator channels have the following spectral responses: $695 \mathrm{~nm}<$ channel $1<700 \mathrm{~nm}, 700 \mathrm{~nm}$ $<$ channel $2<714 \mathrm{~nm}, 714 \mathrm{~nm}<$ channel $3<754 \mathrm{~nm}$, and $754 \mathrm{~nm}<$ channel $4<880 \mathrm{~nm}$. Inside the polychromator, the collected light travels toward the first interference filter where some of the light is transmitted and detected by an avalanche photodiode (channel 1), while some is reflected onto the second channel and so on, until the fourth channel. The optical path inside the polychromator from channel 1 to channel 4 is about $2 \mathrm{~m}$. Table I presents the length of each fiber and respective radial position of observation inside the plasma for the actual setup. Figures 2 and 3 show typical temperature and density profiles for $4.5 \mathrm{~ms}$ during the discharge. The maximum electron temperature and density was around $130 \mathrm{eV}$ and $1.6 \times 10^{19} \mathrm{~m}^{-3}$, respectively. The error bars represent the deviation of the plasma parameters in five discharges of the machine. In a Thomson scattering diagnostic the concern about the stray light starts during the project with the compromise to keep the laser 


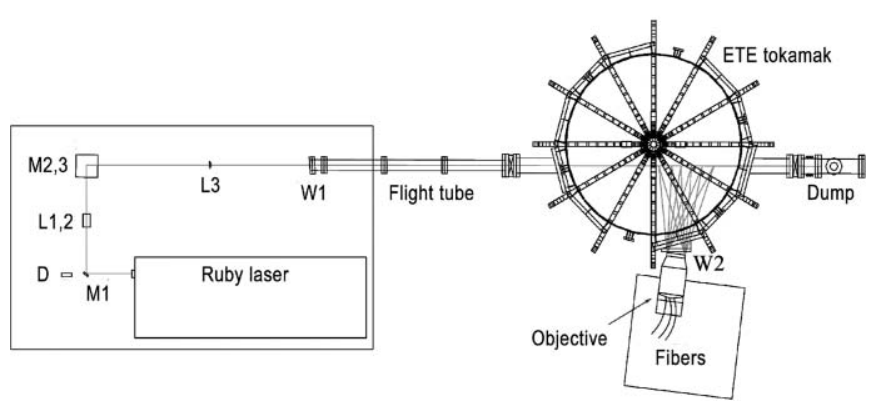

FIG. 1. Drawing of the Thomson scattering diagnostic. M1, 2, 3: flat mirrors; L1, 2: beam expander; L3: focusing lens; D: photodiode; W1: entrance window; W2: collection window.

beam far from the surfaces and at the same time introducing vanes to block the stray light generated by the optical surfaces. Figure 4 shows the present stray light level in the 23 possible fiber positions. All the stray light measurements were done in vacuum, keeping the same value of the parameters used during the temperature and density measurements. Channels 1 and 2 of the polychromator show stray signals mainly from the radial positions at 16.5 and $31.2 \mathrm{~cm}$ with intensity up to $40 \mathrm{mV}$. The most sensitive channels ( 3 and 4 ) of the polychromator show stray signals from several radial positions with a peak of $470 \mathrm{mV}$ at $16.5 \mathrm{~cm}$. As seen in Fig. 4, there is a large variation in the stray light level for the different polychromator channels at a given radial position. This effect is related to the channel sensitivity. The farther the spectral band of the channel from the ruby laser line, the more sensitivity it is. This increase in the sensitivity is to compensate the low plasma scattering signal for distant channels from ruby laser line. The errors on these measurements are less than $10 \%$ and are mainly related with the variation on the intensity of the laser beam in different discharges since the measurements were carried out in vacuum. For this level of noise, the plasma scattered signals from some positions in the present system are masked by the stray light. In principle, the improvement of the signal to noise ratio could be done by two ways: (a) By increasing the energy of the laser, but in our case, as the laser is firing near the threshold density energy of the optical components this could damage the optics. (b) By enlarging the observation region inside the plasma, but that would involve the acquisition of a new polychromator, objective, and

TABLE I. Fiber length and radial positions of observation of each fiber.

\begin{tabular}{lcc}
\hline \hline Fiber no. & Fiber length $(\mathrm{m})$ & Radial positions of observation $(\mathrm{cm})$ \\
\hline 1 & 8 & 44.4 or 46.3 \\
2 & 22 & 20.6 or 21.7 \\
3 & 36 & 39.7 or 42.2 \\
4 & 50 & 17.6 or 19.1 \\
5 & 64 & 31.2 or 33.8 \\
6 & 78 & 35.6 or 37.8 \\
7 & 92 & 23.5 or 25.7 \\
8 & 106 & 27.5 or 29.4 \\
9 & 120 & 48.5 or 51 or 52.9 or 55.1 \\
10 & 134 & 15.1 or 15.8 or 16.5 \\
\hline \hline
\end{tabular}

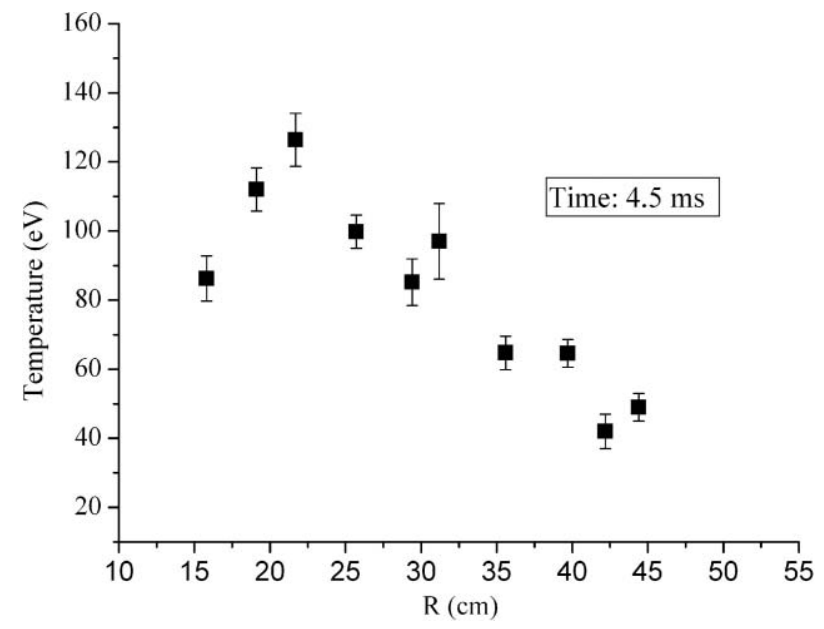

FIG. 2. Temperature profile for $4.5 \mathrm{~ms}$ during the discharge.

fibers. In both cases above, there is no warranty that the stray light will not increase proportionally. In the case of ETE, we decided to do a detailed analysis of the stray light to identify the hot points and afterward to implement changes improving the signal to noise ratio.

\section{BIDIRECTIONAL REFLECTANCE DISTRIBUTION FUNCTION MEASUREMENTS}

In the simulation of the Thomson scattering components it would be possible to use different reflection and absorption coefficients and a Lambertian scattering profile to describe the non optical surfaces like steel with and without black paint, inconel, anodized aluminum, and graphite. In order to have near realistic results, the scattering profiles of these surfaces were measured in the laboratory. The bidirectional reflectance distribution function (BRDF) defined as the ratio of reflected radiance $\left(\mathrm{W} \mathrm{m}^{-2} \mathrm{sr}^{-1}\right)$ at polar angle $\Theta_{s}$ to the incident irradiance $\left(\mathrm{W} \mathrm{m}^{-2}\right)$ on the surface at polar angle $\Theta_{i}$ describes how light is reflected on an opaque surface., ${ }^{4,5}$ Since the measurements were performed on a flat surface, the azimuthal incident and scattered angles were equal to zero. Figure 5 shows the set up used in these measurements. The light source is a $1000 \mathrm{~W}$

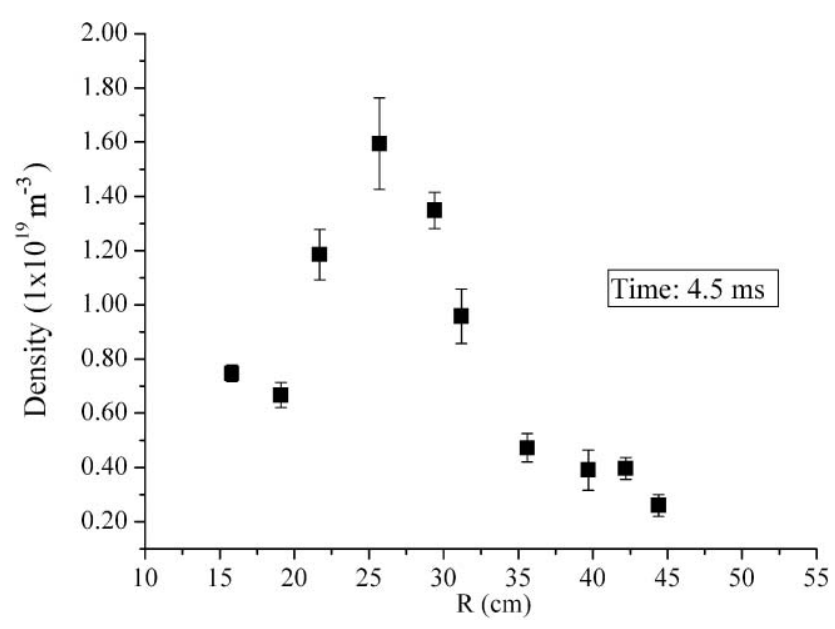

FIG. 3. Density profile for $4.5 \mathrm{~ms}$ during the discharge. 


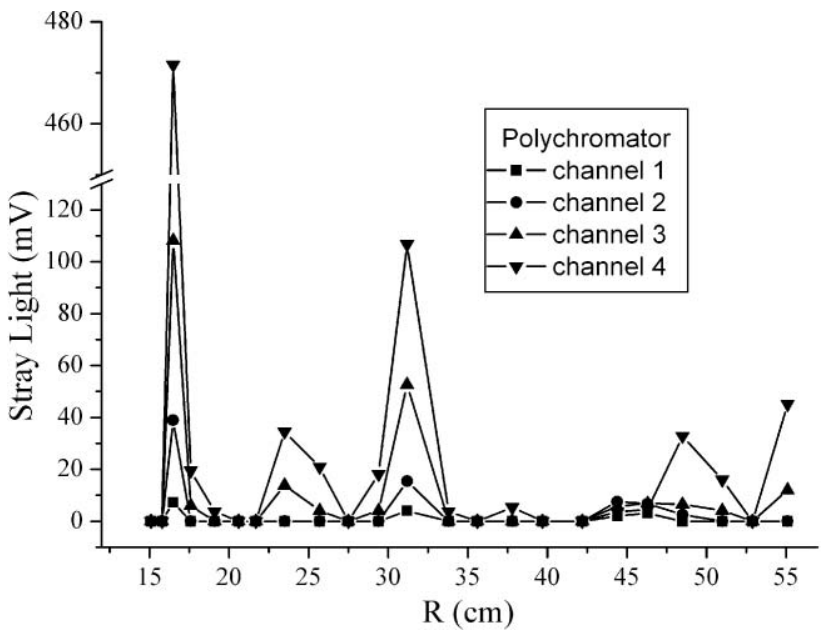

FIG. 4. Stray light profile for the present Thomson scattering setup. The errors of the measurements are less than $10 \%$.

quartz-halogen tungsten filament lamp (Newport 6315) with a specially designed asphere optics to produce a collimated beam of $50 \mathrm{~mm}$ in diameter and divergence less than $22 \mathrm{mrad}$. The samples are placed $1 \mathrm{~m}$ far from the lamp and a rotation stage was used to rotate the samples with an accuracy of $1^{\circ}$. A reflex telescope (Optronic Laboratories-OL 730 9Q) attached to a spectroradiometer (Optronic Laboratories model 740A) composed of a f/4 Czerny-Turner monochromator and a silicon detector radiometer (Optronic Laboratories model 730A) was used to measure the spectral radiance reflected from the samples surface. All the measurements were carried out at $694.3 \mathrm{~nm}$ ruby wavelength using a $3 \mathrm{~mm}$ slit at the entrance of the monochromator resulting in a dispersion

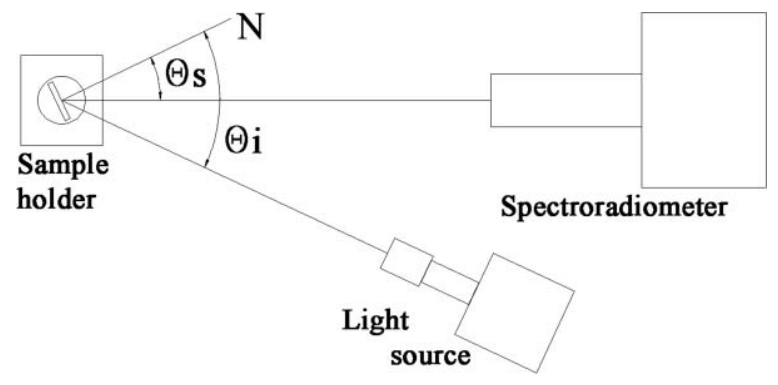

FIG. 5. Set up for BRDF measurements. $\Theta_{i}$ and $\Theta_{s}$ are the incident and the scattered angles, respectively, and $\mathrm{N}$ is the normal to the sample surface.

of $12 \mathrm{~nm}$. With this telescope and slit, the measurements were averaged over an area of $30 \mathrm{~mm}$ diameter on the samples surface. All the samples were $50 \mathrm{~mm}$ in diameter and $10 \mathrm{~mm}$ in thickness and the surfaces were prepared to have almost the same finishing of the parts used in the diagnostic. A standard reflectance from GetSpec (GetReflex) was used to determine the irradiance on the samples surface for each incident angle of the light beam. For each sample, the incident angle $\left(\Theta_{i}\right)$ of the light source was increased from $-60^{\circ}$ to $80^{\circ}$ with steps of $10^{\circ}$. As the spectroradiometer was fixed on the table, the measured scattered angle $\Theta_{s}$ was determined by the relative angular position of the samples and light source, which limited the measurements for some incident angles. For this reason and due to the limitation of the assembly, the number of scattered measured points varied between 5 and 20 for each sample. The step between measured points was $5^{\circ}$. Figure 6 shows an example of the BRDF measurements for five samples for an incident angle of $10^{\circ}$. The figure shows that the steel and inconel samples describe near specular
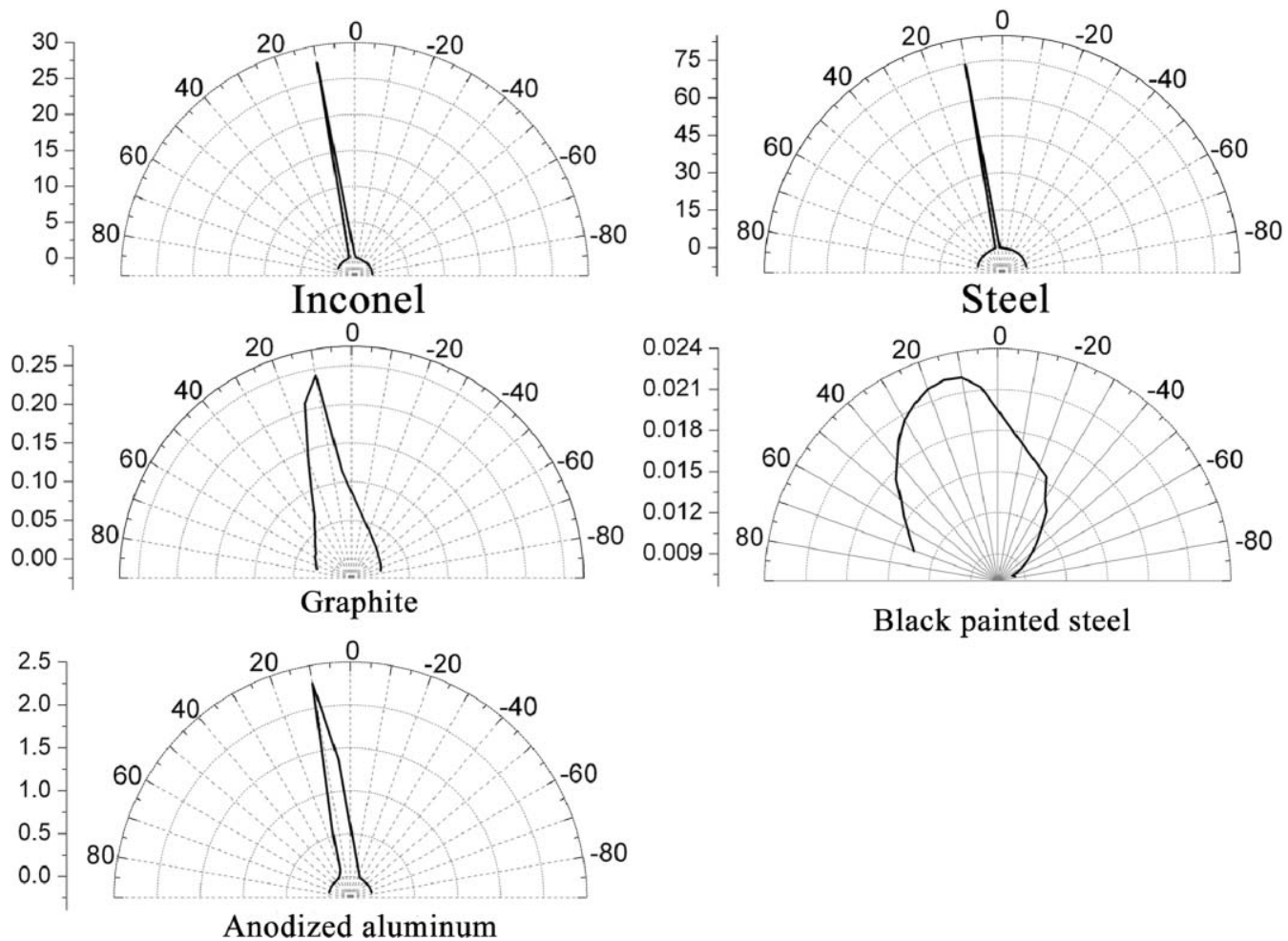

Black painted steel

FIG. 6. Polar plot for the measured profiles for an incident beam at $10^{\circ}$. 


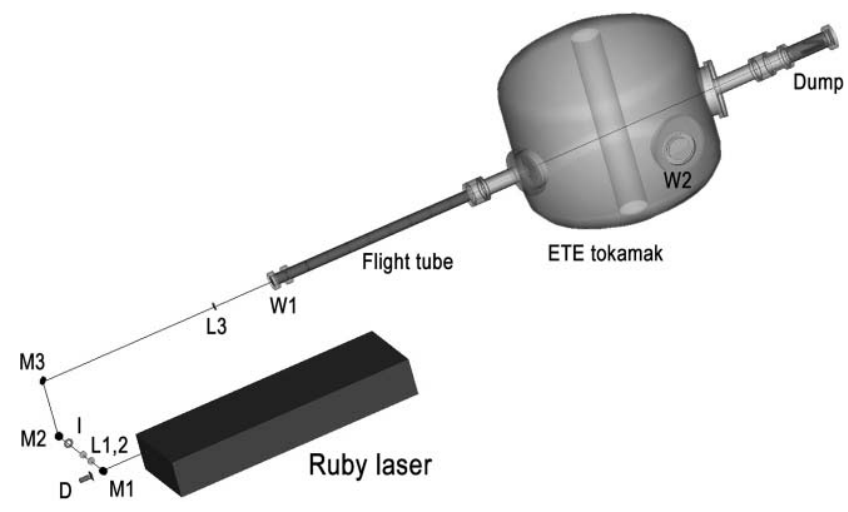

FIG. 7. 3D CAD design used in the ZEMAX simulation.

behavior, the graphite and anodized aluminum describe narrow scattering profiles, and the black painted sample a broad profile.

\section{RAY-TRACING SIMULATION}

To perform a detailed stray light analysis, the threedimensional mechanical CAD model of the diagnostic was transferred to ZEMAX ${ }^{6}$ using SAT (Standard ACIS Text) routines. All the optical components (lenses and windows) were modeled using ZEMAX optical surfaces. Figure 7 shows the whole set up used in the ZEMAX simulation. In this figure the scattered rays were ignored to provide a clear view of the set up and the beam path through the system. Some simplifications were considered in this step: not all vessel windows were drawn, just the windows used in this diagnostic, the outside vessel ETE supports of optics components, and others diagnostics were not considered. The properties of the optical components were provided by the manufacturers and the measured reflectance distribution functions were used for the non optical surfaces. The vane (I) made of anodized aluminum after the beam expander is used to avoid some stray light reaching the mirrors $\mathrm{M} 2,3$. The inner walls of the flight tube and dump are revested by sheets of black painted stainless steel. The vanes inside the flight tube and dump are made

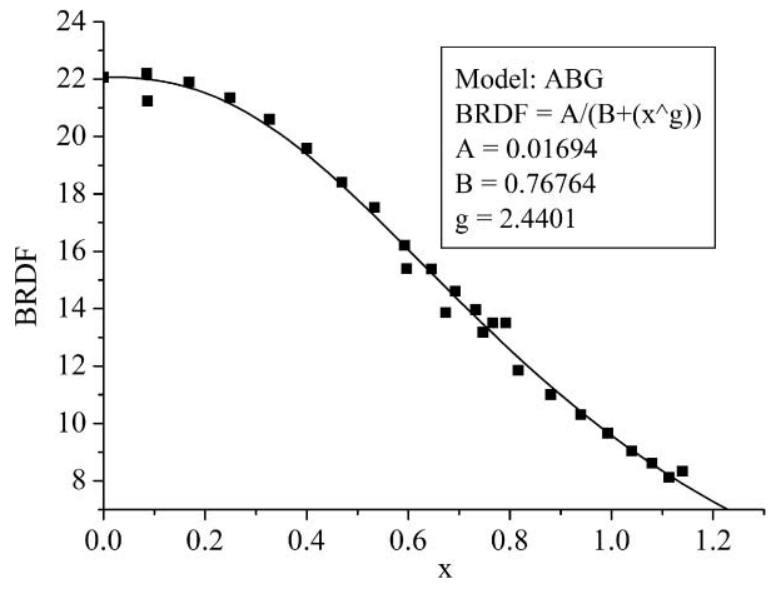

FIG. 8. $A B g$ model fitting for black painted steel sample at an incident angle of $10^{\circ}$. In the horizontal axis $x=\left|\vec{\beta}-\vec{\beta}_{0}\right|$.

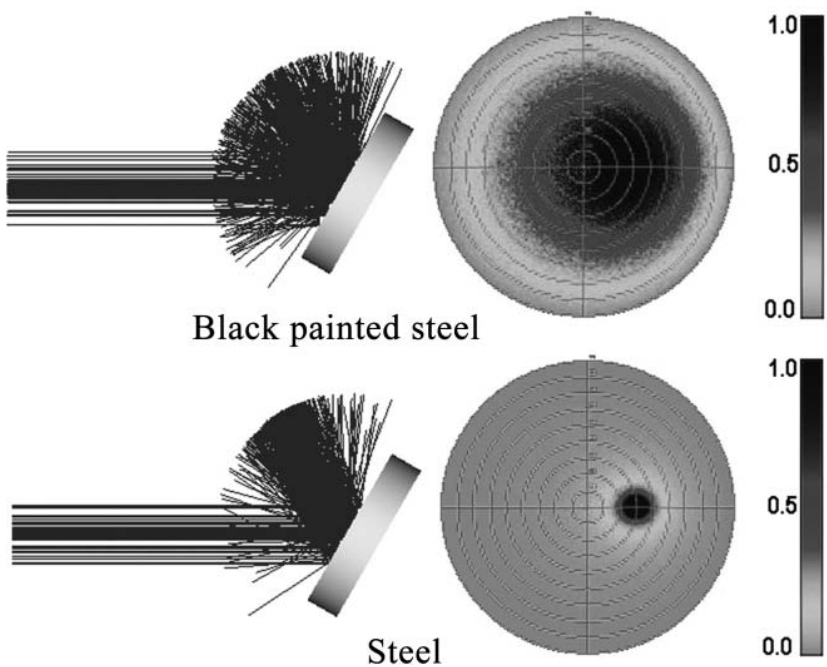

FIG. 9. Scattered polar profiles for an incident beam at $30^{\circ}$ for steel and black painted steel samples simulated in ZEMAX using the $A B g$ model for the measured data. The vertical bar indicates the relative intensity of the scattered light from the sample surface.

of black painted stainless steel. Inside the dump there are two graphite plates to stop the laser beam and the horizontal plate has special design to minimize the stray light. The vacuum vessel of ETE is made of inconel and all the vacuum connections, valves, and windows ports are made of stainless steel. In the ZEMAX program, the BRDF profiles can be described by four models-Lambertian, $A B g$, Gaussian, and user-defined model. In this work, the $A B g$ model was chosen because of good fitting with the measured data as it can be seen in Fig. 8. The $A B g$ BRDF is given by: ${ }^{6,7}$

$$
\operatorname{BRDF}(\vec{x})=\frac{A}{B+|\vec{x}|^{g}},
$$

where, $\vec{x}=\vec{\beta}-\vec{\beta}_{0}, \beta_{0}$, and $\beta$ are the projection of the specular and scattered ray vectors down to the surface, respectively. The coefficients $A, B$, and $g$ are determined by fitting the $A B g$ curve to the measured points and transferred to ZEMAX in appropriate table form. Figure 9 shows the simulated scattering profiles for two test samples using the $A B g$ model. In this figure it is possible to see the difference between the stainless steel with and without the black paint.

\section{A. Stray light simulation of the actual Thomson scattering system}

In the simulation, the laser beam energy was fixed at $10 \mathrm{~J}$ with a diameter of $19 \mathrm{~mm}$ and a divergence of $1 \mathrm{mrad}$. The beam is composed of 20000 rays, each ray has $0.5 \mathrm{~mJ}$ and the ray is no longer tracked when its relative energy is less than $1 \times 10^{-8} \mathrm{~J}$ or when absorbed by the walls. During the simulation, two detectors were used to measure the stray light, one of them was set in front of the dump to measure the light coming back from the dump and the other at collection window (W2 in Fig. 7) to measure the stray light that reaches this window directly. With a third detector, set at the focusing point inside the vessel, an energy of $8.2 \mathrm{~J}$ was measured, which indicates a loss of $18 \%$ in the delivery system of the laser pulse. This 


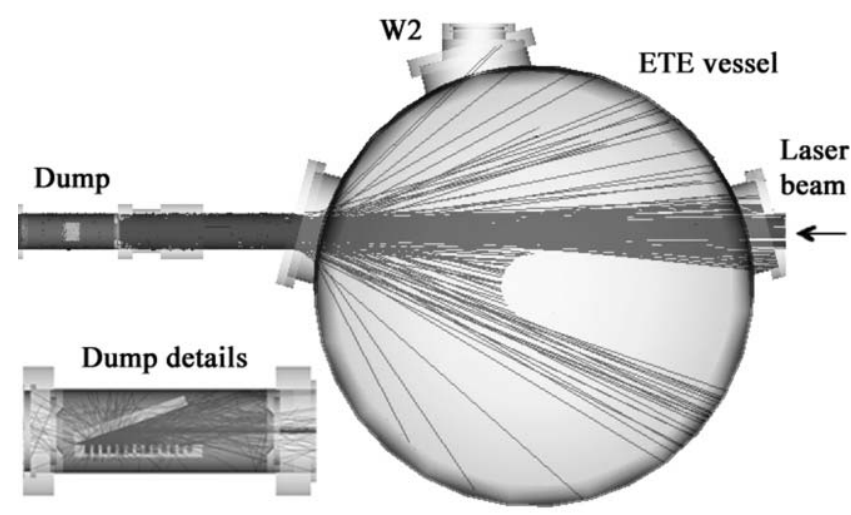

FIG. 10. Details of the scattered light by the graphite dump.

lost energy was absorbed, reflected or scattered in the path between laser and the plasma center. Figure 10 shows details of the inner parts of the dump and how the back scattered light from dump is spread inside the vessel. It is possible to see that some rays reach straight the collection window and that there is a divergent beam toward the port of the flight tube. In this figure, the number of rays was drastically reduced to better visualize the scattered rays. The intensity of this back scattered light from dump is about $1.7 \times 10^{-4} \mathrm{~J}$. From this energy, just $3.3 \times 10^{-6} \mathrm{~J}$ reaches the collection window. Part of this stray light that reaches the collection window is collected by the objective and transmitted into the polychromator through the fibers. In the simulation, it was possible to track the sources of this stray light that reaches the collection window: less than $1 \%$ come straight from the dump, $10 \%$ from reflections on the center column of the vessel and $89 \%$ from the remainder of the vessel.

\section{B. Stray light simulation of the upgrade system}

In the project of the ETE machine, the center column will be covered around by graphite plates in the near future. As the center column contributes $10 \%$ of the stray light that reaches the window, when it was considered in the simulation this graphite coating decreased the stray light at window by two times. Considering the center column with graphite and the same covering on the surface of the conflat port (CF14 in.) of the flight tube that faces the dump the intensity of the stray light decreased by five times. As presented in the previous section, the biggest contribution to the stray light is due to

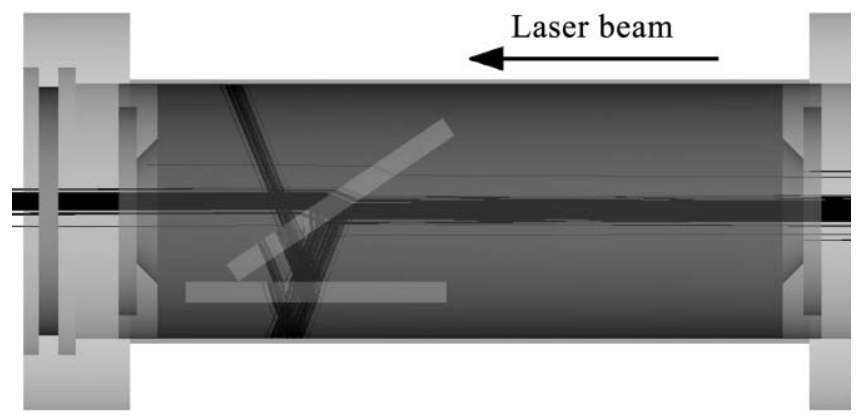

FIG. 11. Beam path inside the blue glass dump.

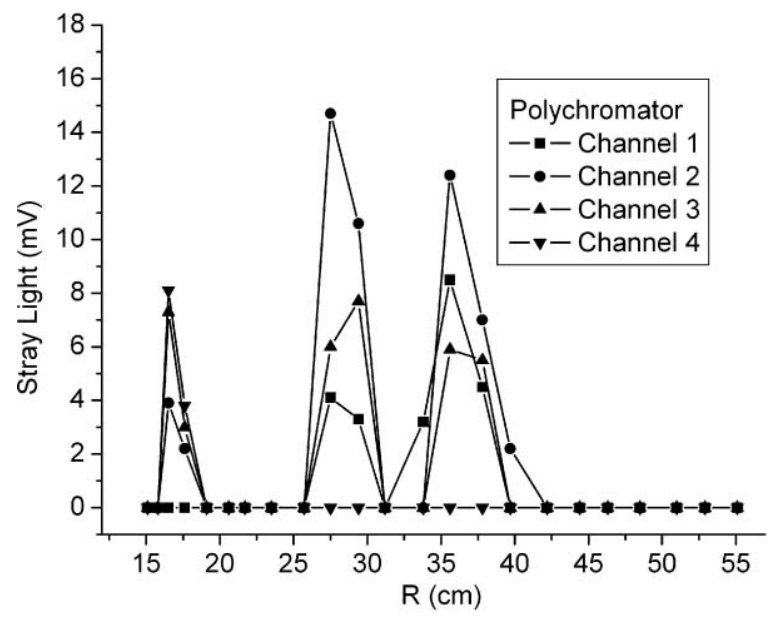

FIG. 12. Stray light profile for the upgraded Thomson scattering setup. The errors of the measurements are less than $10 \%$.

multiple scattering on the walls of the vessel, which is difficult to mitigate. To promote a main reduction of this stray light a new dump was projected. The main task of the project was to use optical glasses to absorb the laser beam and to control the path of the beam inside the dump avoiding back scattering like in the old dump. Because of the mechanical limitations it is not possible to take the new dump far from the actual position and since the energy density of the laser beam inside the dump can reach up to $18 \mathrm{~J} / \mathrm{cm}^{2}$ for the maximum laser energy, the challenge was to find glasses that could absorb this energy without breaking. Using the laser beam several glasses were tested. From usual glasses, that can be found in any glass shop, special glasses like the ones used in welding to protect from UV radiation and, in ovens windows, to some optical glasses. The temperate borosilicate glass with cobalt impurities that gives it a blue color was chosen. ${ }^{8}$ This affordable glass was the best-tested glass for the application. It supported up to $14 \mathrm{~J} / \mathrm{cm}^{2}$ for the same beam area expected inside the dump. This energy threshold of the glass limited the laser energy to $8 \mathrm{~J}$ that is more than what is usually used during the experiment. The new proposed dump uses two temperate blue glass plates with dimensions of $40 \mathrm{~mm} \times 100 \mathrm{~mm}$ and thickness of $8 \mathrm{~mm}$. The first laser impact occurs in one of the plates in Brewster angle that absorbs $80 \%$ of the energy and reflects less than $1 \%$. The second plate is responsible for absorbing the reflection of the first one. These values were measured using the ruby laser beam and a photodiode to detect the transmitted and reflected laser beam incident on the plate at Brewster angle. Figure 11 shows the geometry and the beam path inside the new dump. The simulation with this new dump showed that the stray light at the collection window decreased by 95 times.

\section{RESULTS AND DISCUSSION}

The proposed dump was built and installed at the ETE machine and a new profile of the stray light was measured as shown in Fig. 12. For the first channel of the polychromator a stray light signal of $8 \mathrm{mV}$ was measured at the radial position $35.6 \mathrm{~cm}$ and for the second channel $15 \mathrm{mV}$ at 
$27.5 \mathrm{~cm}$, it shows a reduction of about three times for lesser sensitive channels. Channels 3 and 4 show a reduction of 60 times for the stray light signals with a peak intensity of $8 \mathrm{mV}$ at $16.5 \mathrm{~cm}$. Stray light between positions 22.5 and $25 \mathrm{~cm}$ and after position $42.5 \mathrm{~cm}$ disappeared and new points of stray signal appeared at positions 27.5 and $37.5 \mathrm{~cm}$. With this new dump the reflected back light inside the vessel presents a new distribution which explains the new scattering points as seen in Fig. 12. In Fig. 4, channel 4 shows the largest signals, while in Fig. 12 the largest signals were measured by channel 2 . This disparity can be explained by the difference in the optical path of the light for each fiber inside the polychromator. Since this polychromator was projected just for only one channel using a fiber bundle of $3 \mathrm{~mm}$ in diameter, as detailed in Ref. 9, when the system was upgraded to ten spatial channels, the fibers were accommodated as near as possible to the optical axis of the polychromator. This raises the signal loss because part of the light can stay out of the field of view of the channel detector due to divergence of the light as the fiber is set far from the optical axis. This loss is more sensitive as the spectral channel is far from the polychromator input. The largest signals measured in Fig. 12 are from different radial positions in the vessel and different fibers. The higher reduction expected in the simulation (about 95 times) can be explained by the simplifications that were considered in the 3D model as explained before. Just the total stray light that reached the collection window was estimated and no consid- eration about each fiber and polychromator optics was taken into account.

During the tests to choose the glass for the new dump it was verified that some glasses showed better absorption but they do not support the actual energy density of the laser. In a next opportunity it will be interesting to study the possibility to take the dump far from the actual position decreasing this density to allow the use of other glasses.

\section{ACKNOWLEDGMENTS}

This work has been partially funded by Rede Nacional de Fusão (RNF / FINEP). L. A. Berni would like to thank all the members of ETE tokamak group for their support.

${ }^{1} \mathrm{~J}$. Sheffield, Plasma Scattering of Electromagnetic Radiation (Academic, London, 1975).

${ }^{2}$ L. A. Berni, M. P. Alonso, and R. M. Oliveira, Rev. Sci. Instrum. 75, 3884 (2004).

${ }^{3}$ L. A. Berni, E. Del Bosco, R. M. Oliveira, and M. P. Alonso, Braz. J. Phys. 34, 1572 (2004).

${ }^{4}$ G. J. Ward, Comput. Graph. 26, 265 (1992).

${ }^{5}$ R. Siegal, Thermal Radiation Heat Transfer (Taylor\&Francis, Washington, 1992).

${ }^{6}$ ZEMAX Development Corporation, 2010.

${ }^{7}$ J. E. Harvey and A. Kotha, SPIE 2576, 155 (1995).

${ }^{8}$ Saint Mariane Vidros Especiais Ltda, Brazil, São Paulo.

${ }^{9}$ L. A. Berni, M. Ueda, E. Del Bosco, J. G. Ferreira, R. M. Oliveira, and W. A. Vilela, Rev. Sci. Instrum. 74, 1200 (2003). 